Ariza, T.; Fernández-Jiménez, F.J.; Muñoz-Calle, J.; Sierra, A.J. y Mora, M. (2019) Arquitectura software para la prescripción de ejercicio físico personalizado / Software Architecture for Customized Physical Exercise Prescription. Revista Internacional de Medicina y Ciencias de la Actividad Física y el Deporte vol. 19 (73) pp. 137-150 Http://cdeporte.rediris.es/revista/revista73/artarquitectura985.htm DOI: http://doi.org/10.15366/rimcafd2019.73.010

\title{
ORIGINAL
}

\section{ARQUITECTURA SOFTWARE PARA LA PRESCRIPCIÓN DE EJERCICIO FÍSICO PERSONALIZADO}

\section{SOFTWARE ARCHITECTURE FOR CUSTOMIZED PHYSICAL EXERCISE PRESCRIPTION}

\author{
Ariza, T.1; Fernández-Jiménez, F.J. ${ }^{2}$; Muñoz-Calle, J. ${ }^{3}$ Sierra, A.J. ${ }^{3}$ y Mora, M. ${ }^{4}$ \\ ${ }^{1}$ Doctora en Informática, Departamento de Ingeniería Telemática, Universidad de Sevilla (España) \\ teresa@trajano.us.es \\ 2 Ingeniero de Telecomunicación, Departamento de Ingeniería Telemática, Universidad de Sevilla \\ (España) fjfj@trajano.us.es \\ ${ }^{3}$ Doctores en Ingeniería de Telecomunicación, Departamento de Ingeniería Telemática, Universidad \\ de Sevilla (España) javi@trajano.us.es, antonio@trajano.us.es \\ 4 Doctora en Medicina y Ciencias Aplicadas al Deporte, Departamento de Educación Física y \\ Deporte. Universidad de Sevilla (España) matimora@us.es
}

\section{AGRADECIMIENTOS}

Este trabajo no habría sido posible sin la dedicación y el entusiasmo de los alumnos de proyectos fin de carrera de Ingeniería de Telecomunicación y trabajos fin de Grado en Tecnologías de las Telecomunicaciones de la Escuela Superior de Ingeniería de la Universidad de Sevilla A. J. Díaz, J. García, P. Gallegos y J. M. Valverde, que ha permitido la construcción del prototipo.

Código UNESCO / UNESCO code: 330499 Otras: eSalud/ Others: eHealth Clasificación Consejo de Europa / Council of Europe classification: 17 Otras: eSalud/ Others: eHealth

Recibido 15 de febrero de 2016 Received February 15, 2016 Aceptado 26 de julio de 2017 Accepted July 26, 2017

\section{RESUMEN}

En la actualidad existe una gran cantidad de aplicaciones para la realización de ejercicio físico. En este artículo se presenta una arquitectura software para una aplicación que permite la prescripción de ejercicios físicos personalizados. Esta 
arquitectura incluye dos funcionalidades, la funcionalidad para el especialista que prescribe el ejercicio y la funcionalidad para el usuario que debe seguir esta prescripción. La interacción entre el especialista y el usuario se realiza mediante el envío de eventos. Especialistas (médicos/fisioterapeutas/educadores físicos) pueden indicar los ejercicios adecuados en cada caso. Esta aplicación permitirá a los usuarios con demanda de atención personalizada mejorar la condición física y la calidad de vida. Los usuarios podrán realizar el ejercicio físico de forma autónoma, sin tener que realizar desplazamientos y en un horario flexible.

PALABRAS CLAVE: eSalud, Programación de Dispositivos Móviles, Ejercicio Físico, Prescripción de ejercicio, Sistema Software.

\section{ABSTRACT}

Currently there is a lot of available applications to do physical exercise. This article describes the software architecture for an application that allows customized exercise prescription. This architecture includes two functionalities, the functionality that allows specialists to prescribe physical exercises and the functionality for users who must follow this prescription. Interactions between specialists and users are allowed by means of sending events. Specialists (doctors, physiotherapists and physical educators) can indicate appropriate exercises in each case. This application will allow users with demand for personalized attention to improve their fitness and quality of life. Users can perform physical exercise autonomously, without having to travel and in a flexible schedule.

KEYWORDS: health information systems, mobile computing, physical exercise, prescription, software system.

\section{INTRODUCCIÓN}

En la actualidad existen multitud de estudios que investigan sobre la salud y la influencia de la actividad física en la mejora de la calidad de vida de las personas. Desde las instituciones públicas y privadas se están desarrollando programas de ejercicios en relación con la salud, dirigidos a diferentes colectivos. En los últimos años, quizás la mayor parte de estos programas están orientados a personas mayores y grupos de población con alguna necesidad específica (diabetes, hipertensión, obesidad, fibromialgia, cáncer, depresión...) [1][2]. La población en general demanda una actividad física saludable. Para sentirse mejor y poder realizar actividades de la vida diaria con un menor esfuerzo y gasto energético, existe una necesidad de hacer ejercicio físico. A su vez, constituye un grave problema el desconocimiento de una adecuada prescripción del ejercicio y el asesoramiento de personas no especializadas en este ámbito [3]. Cuando utilizamos "la actividad física" como herramienta terapéutica y preventiva, entonces debemos hablar de 
prescripción de ejercicio físico que se define [4] como el "proceso mediante el cual se recomienda a una persona un régimen de actividad física de manera sistemática e individualizada, para obtener los mayores beneficios con los menores riesgos". La eficacia de los programas de ejercicio físico está perfectamente demostrada en el tratamiento y prevención de numerosas situaciones.

La práctica de actividad física constituye uno de los pilares fundamentales de un estilo de vida saludable y de una verdadera protección y promoción de la salud. La actividad física regular asegura, a las personas de todas las edades, unos beneficios evidentes para su salud física, social y mental, así como para su bienestar general [5]. El objetivo del ejercicio variará según el colectivo y sus características, pero el término salud, implica una mejora en la capacidad funcional del sujeto (resistencia aeróbica, fortalecimiento muscular y flexibilidad) y aspectos psico-sociales (autoestima, ansiedad, relaciones, imagen corporal, ...) [6].

La expansión de las tecnologías de la información y las comunicaciones a todos los ámbitos de nuestra vida (profesional, ocio, cuidado personal, ...) ha permitido familiarizar a toda la población con las nuevas tecnologías y que sea algo más o menos cotidiano en la vida de la mayoría de las personas. Por otro lado, el abaratamiento de los dispositivos informáticos y de telecomunicación (ordenadores, móviles, tabletas, impresoras, ...) ha constituido un hecho fundamental en el acceso a estas tecnologías por parte de la población en general [7].

Aunque existe una gran cantidad de aplicaciones móviles para realizar ejercicio físico, como Runstatic, Endomondo.... que están pensadas para personas sanas, que quieren realizar un plan de entrenamiento, estas aplicaciones no están adaptadas para colectivos con características especiales que necesitan realizar ejercicio físico, ya sea como prevención o como mejora de determinados problemas en relación con el aparato locomotor (artrosis, dolores musculares...), cardiovascular (hipertensión) o metabólicos (diabetes, colesterol, obesidad...). Por otra parte, hay herramientas para la prescripción de ejercicio [8] conteniendo bases de datos con un gran número de ejercicios que permiten al especialista prescribir ejercicio a los usuarios. Los ejercicios prescritos habitualmente se imprimen o se mandan por correo electrónico al usuario. Sin embargo, supone una mejora la realización de estos ejercicios usando dispositivos móviles. Los usuarios podrán realizar el ejercicio físico de forma autónoma, sin tener que realizar desplazamientos y en un horario flexible. Esto permitiría a usuarios con demanda de atención personalizada mejorar la condición física y la calidad de vida.

En el resto del artículo se presentan los objetivos del trabajo, la arquitectura del sistema propuesto, detallando los distintos componentes de la arquitectura, el prototipo realizado y las conclusiones del trabajo. 


\section{OBJETIVOS}

El objetivo de este trabajo es ayudar a la prescripción de ejercicios físicos de forma personalizada, para personas mayores o poblaciones con necesidades específicas, que podrá ser guiado por un equipo multidisciplinar integrado por médicos, psicólogos y profesionales de ciencias de la actividad física. Para ello se propone una arquitectura software de referencia para una aplicación telemática. Se realizará el análisis y el diseño, así como la implementación y prueba de un prototipo de la aplicación que permita la prescripción de ejercicios físicos personalizados a cada persona.

Lo que se conseguirá con esta aplicación es poner a disposición del personal especializado una forma más cómoda de indicar a sus pacientes los ejercicios adecuados y recomendados para mejorar su dolencia o simplemente para mejorar su condición física y con ello su calidad de vida. También ayudará a los usuarios a la realización de estos ejercicios, siempre bajo las recomendaciones del especialista, sin necesidad de desplazamientos ni sujetos a horarios impuestos mediante el uso de una aplicación móvil.

\section{MATERIAL Y MÉTODOS}

Para llevar a cabo los objetivos marcados en la sección anterior se propone una arquitectura software para la aplicación de la prescripción de ejercicio físico personalizado. La funcionalidad se puede ver desde el punto de vista del especialista que prescribe el ejercicio físico o desde el punto de vista del usuario que debe realizar estos ejercicios para mejorar su condición física.

El especialista puede prescribir ejercicios físicos a los usuarios de la aplicación de forma personalizada. Para ello, el sistema debe tener la información necesaria acerca de los usuarios y de los ejercicios que puede prescribir el especialista, así como de las rutinas de entrenamiento entendidas como un conjunto de ejercicios a realizar por el usuario. El sistema permitirá dar de alta a nuevos usuarios y nuevos ejercicios, así como asignar ejercicios a las rutinas de entrenamiento y asignar rutinas a un usuario.

Desde el punto de vista del usuario, éste podrá visualizar los ejercicios físicos asignados por el especialista de una forma cómoda mientras los realiza. Para ello tendrá disponible una aplicación móvil que le permitirá autenticarse en el sistema si ya está registrado o bien registrarse en caso de que aún no esté dado de alta, y acceder a todas las rutinas de entrenamiento asignadas, visualizando los ejercicios que debe realizar y pudiendo consultar sus descripciones.

Para dotar de estas funcionalidades a la aplicación, la arquitectura propuesta se divide en cuatro subsistemas diferentes: 
- $\quad$ Subsistema de gestión de la base de datos: encargado del manejo de los datos de usuarios, ejercicios y rutinas necesarios para la prescripción de ejercicios.

- $\quad$ Subsistema de eventos: Permite almacenar eventos en una cola de eventos y recuperarlos desacoplando a los productores y consumidores de eventos (usuarios y especialistas respectivamente).

- Subsistema de prescripción para el personal especializado: aplicación que permite al personal especializado indicar los ejercicios prescritos a cada usuario que vaya a usar la herramienta. Utiliza el subsistema de gestión de la base de datos para el acceso a los datos y el subsistema de eventos para el acceso a los eventos producidos por los usuarios.

- Subsistema de usuario: aplicación usada por el usuario final para la realización de los ejercicios prescritos por el personal especializado. Utiliza el subsistema de gestión de la base de datos para el acceso a los datos y el subsistema de eventos para el envío de eventos producidos por los usuarios.

La ilustración 1 muestra esta arquitectura con los 4 subsistemas y la interacción con el especialista y el usuario.

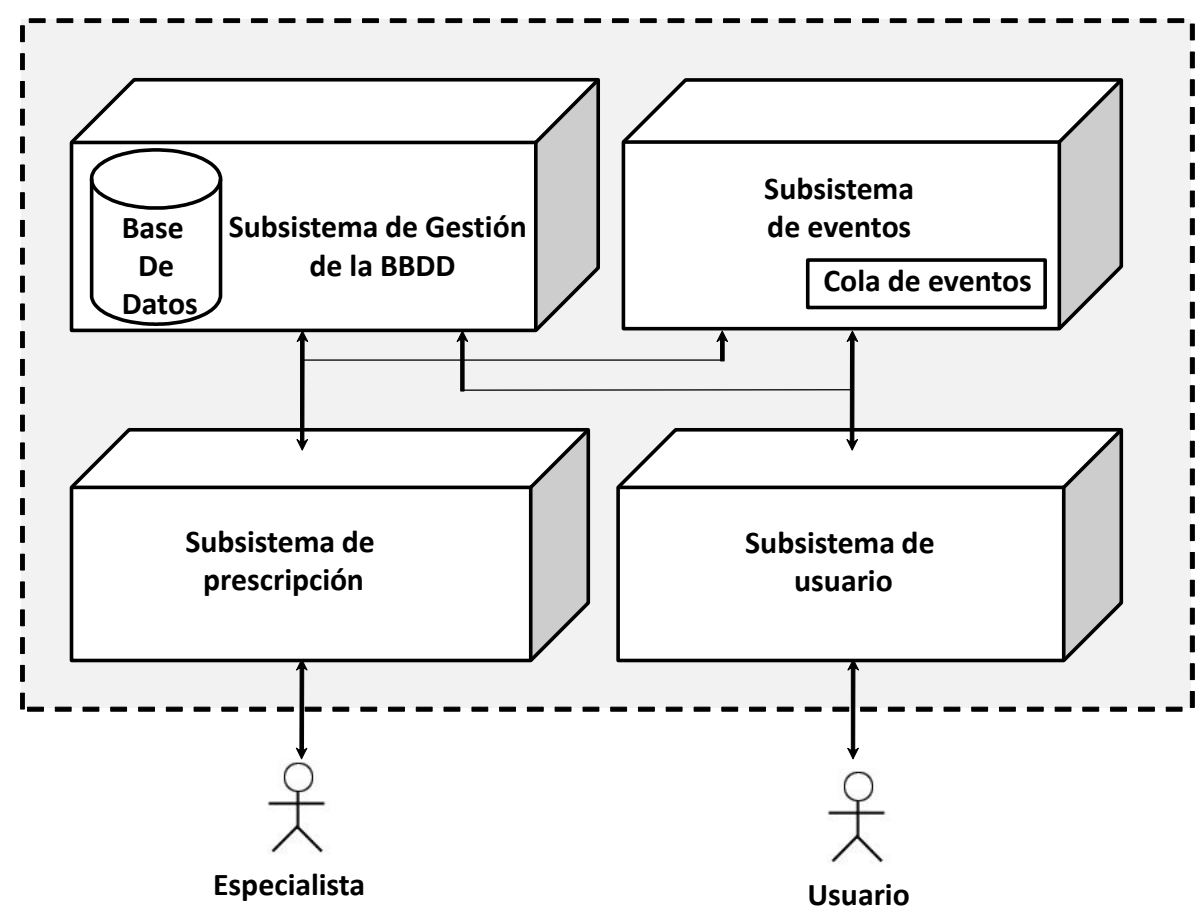

Ilustración 1. Arquitectura del sistema 
En los siguientes apartados se detallan los distintos subsistemas y el prototipo desarrollado.

\section{SUBSISTEMA DE GESTIÓN DE BASE DE DATOS}

El subsistema de gestión de base de datos se encarga del mantenimiento (creación, lectura, actualización y borrado) de los datos necesarios para la prescripción del ejercicio físico por parte del especialista. En la base de datos se almacenan los datos correspondientes a los usuarios, ejercicios y rutinas asignadas a los distintos usuarios.

El esquema de la base de datos usada es el mostrado en la ilustración 2. Las principales tablas de la base de datos son las siguientes:

- Usuarios: Almacena los datos principales de los usuarios, tales como nombre, apellidos, usuario, contraseña necesaria para la autenticación, etc.

- $\quad$ Rutinas: Contiene las diferentes rutinas establecidas por el especialista para asignarlas a los diferentes usuarios.

- Ejercicios: En esta tabla se encuentran los distintos ejercicios físicos junto con su descripción, su finalidad y su duración.

- Útiles: Aquí están contenidos los diferentes útiles que pueden ser necesarios para realizar un ejercicio.

- $\quad$ Recursos: En esta tabla se encontrarán las localizaciones de recursos para la realización del ejercicio tales como fotos o los vídeos de cada ejercicio.

- Músculos: Tabla que almacena información acerca de los músculos que serán trabajados mediante los distintos ejercicios.

- ExplicacionesDeEjercicios: Tabla que contiene una explicación completa de cómo realizar cada ejercicio.

- CaracteristicasDeEjercicios: En esta tabla se encuentran las principales características de un ejercicio, para qué sirve y zonas afectadas.

- RecomendacionesDeEjercicios: En esta última tabla se indican las recomendaciones de cada ejercicio, a quién va dirigido y aspectos a tener en cuenta a la hora de realizar el ejercicio.

Por otro lado, hay tablas que relacionan unas con otras. Estas tablas son:

- RutinasDeUsuarios: Relaciona los usuarios con las rutinas que se le asignan dependiendo de los que decida el especialista.

- EjerciciosDeRutinas: En esta tabla se asignan a cada rutina un número determinado de ejercicios, que serán los que deben hacerse al elegir dicha rutina. 
- MusculosDeEjercicios: Contiene la relación entre un ejercicio y los diferentes músculos que participan en dicho ejercicio.

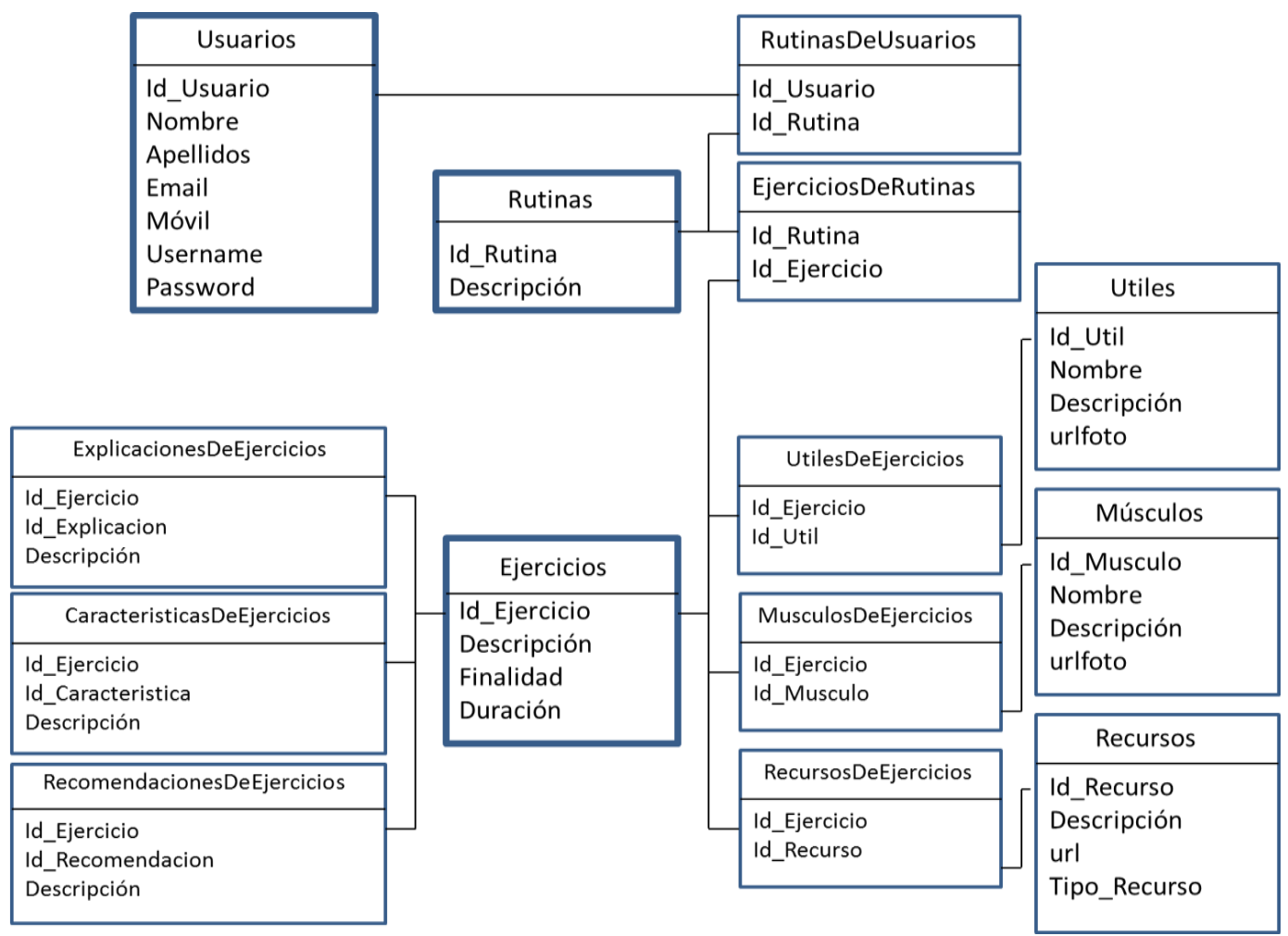

Ilustración 2. Esquema de la Base de Datos para la prescripción de ejercicios

- RecursosDeEjercicios: Esta tabla relaciona un ejercicio con los recursos disponibles para él, tales como las fotos o videos que le corresponden.

- UtilesDeEjercicios: Esta tabla relaciona los útiles disponibles con los ejercicios.

Este subsistema de gestión de base de datos es usado tanto por el subsistema de prescripción como por el subsistema de usuario.

\section{SUBSISTEMA DE PRESCRIPCIÓN}

El subsistema de prescripción es la herramienta que usa el especialista para realizar una gestión personalizada de los usuarios. Esta herramienta, llamada Gestor de Entrenamientos, permite asignar rutinas de ejercicios a un usuario. Se usa a través de una interfaz gráfica intuitiva. Las principales funciones de este subsistema son:

- $\quad$ Crear, modificar y eliminar usuarios. 
- Mandar un correo electrónico en caso de que olvide la contraseña, con la nueva contraseña asignada por el sistema.

- Crear, modificar y eliminar rutinas.

- Crear, modificar y eliminar ejercicios.

- Modificar las rutinas asignadas a cada usuario, permitiendo añadir nuevas o eliminar las antiguas.

- Modificar los ejercicios que contiene cada rutina, permitiendo añadir nuevos o eliminar los antiguos.

- Modificar las fotos y los vídeos que se mostrarán de cada ejercicio en la aplicación del usuario.

- Visualizar los eventos que se han producido por parte de los usuarios.

\section{SUBSISTEMA DE USUARIO}

El subsistema de usuario tiene como objetivo permitir que un usuario pueda visualizar en su dispositivo móvil los ejercicios físicos que el especialista le haya prescrito. Además de esto, permite enviar información acerca de las rutinas que realiza el usuario.

La aplicación tiene diferentes funcionalidades para el usuario. Éste puede acceder a la aplicación una vez registrado en el sistema o bien sin registro. En este último caso, sólo se tendrá acceso a un conjunto limitado de rutinas generales y se tendrá la posibilidad de registrarse en el sistema dando los datos de usuario necesarios para el registro. Si accede un usuario registrado, entonces la aplicación ofrecerá las siguientes funcionalidades:

- Cambio/modificación de la información personal de usuarios registrados.

- Petición de nueva contraseña en caso de olvido vía correo electrónico.

- Acceso a las rutinas del usuario.

- Acceso libre a todas las rutinas.

- Dentro de las rutinas, acceso a todos los ejercicios de esa rutina.

El acceso a los ejercicios se podrá realizar de dos maneras diferentes:

- Acceso detallado a cada uno de los ejercicios.

- Acceso rápido a los ejercicios, mediante una lista con sólo los vídeos de los ejercicios de la rutina a realizar.

Dentro del acceso detallado a cada uno de los ejercicios particulares de los que dispone cada rutina, el usuario podrá:

- Ver la descripción del ejercicio. 
- Ver la finalidad del ejercicio.

- Ver la duración (número de series o repeticiones).

- Acceder a propiedades del ejercicio, características o recomendaciones.

- Ver los útiles (si estos son necesarios) para realizar el ejercicio, tales como cintas elásticas, pesas, esterillas, etc.

- Ver los músculos implicados en el ejercicio, con una descripción y una foto de cada uno de ellos.

- Para una mayor comprensión del ejercicio, fotos del ejercicio a realizar, tales como una foto de la posición inicial del ejercicio, otra foto con la posición final, así como una foto con un detalle del ejercicio que el médico o entrenador ha estimado oportuno resaltar.

- $\quad$ Por último, un video explicativo del ejercicio, donde aparece simultáneamente la realización del ejercicio, así como una explicación.

\section{SUBSISTEMA DE EVENTOS}

El subsistema de eventos permite comunicar eventos producidos en el sistema por el usuario. Por ejemplo, por medio de la cola de eventos se informa al especialista de las rutinas que ha realizado el usuario. También mediante esta cola de eventos el usuario puede pedir que el especialista se ponga en contacto con él para consultarle acerca de los ejercicios. Esto permite la realización de ejercicios de una forma supervisada por el especialista.

En la ilustración 3 se muestra el esquema de la comunicación de eventos entre el usuario y el especialista. Los eventos son producidos por los usuarios y consumidos por los especialistas.

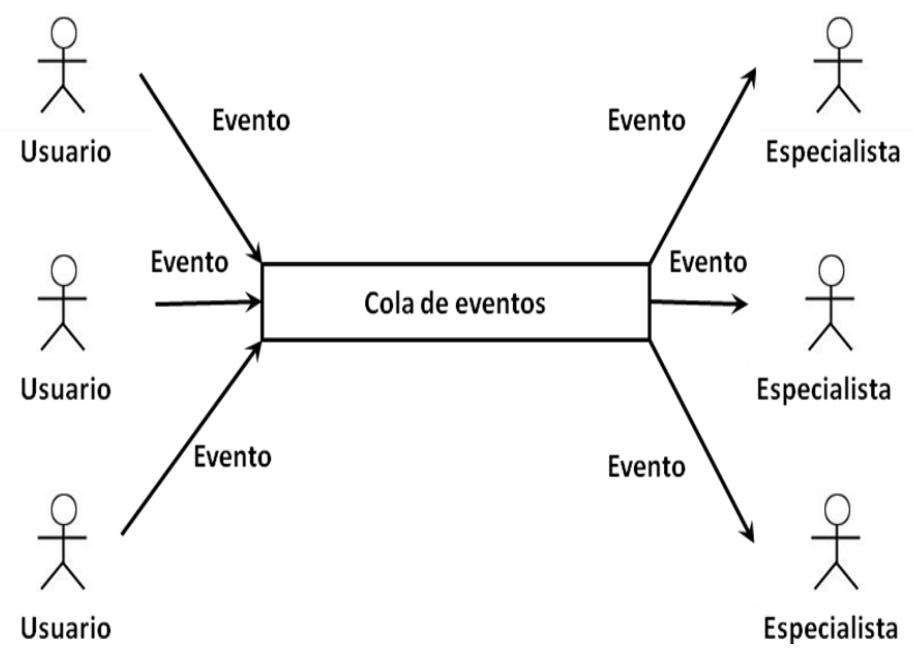

Ilustración 3. Esquema de comunicación de eventos 


\section{PROTOTIPO REALIZADO}

Como parte de este trabajo se ha realizado un prototipo para la prescripción de ejercicios físicos a usuarios por parte del especialista. Se ha desarrollado una aplicación de escritorio para que sea usada por el especialista para la prescripción y una aplicación móvil para que sea utilizada por el usuario, así como el software necesario para el acceso a la base de datos y el acceso a la cola de mensajes.

La arquitectura del prototipo desarrollado, incluyendo las tecnologías usadas en la construcción de éste, se muestra en la ilustración 4.

Las tecnologías usadas en el prototipo son las siguientes:

- Java: Es un lenguaje de programación orientado a objetos, multiplataforma, muy popular para la construcción de aplicaciones distribuidas. Se ha usado para desarrollar la aplicación del especialista.

- $\quad$ Android: Es un sistema operativo para teléfonos móviles. Está basado en el sistema operativo Linux, un núcleo de sistema operativo libre, gratuito y multiplataforma. El sistema operativo proporciona todas las interfaces necesarias para desarrollar aplicaciones que accedan a las funciones del teléfono (como la ubicación, las llamadas, la agenda, etc.) de una forma muy sencilla en el lenguaje de programación Java. Desde hace varios años el sistema operativo Android es el que más cuota de mercado alcanza entre los dispositivos móviles.

- MySQL: Es un sistema de gestión de bases de datos relacional, multihilo y multiusuario. Se utiliza este gestor para el almacenamiento de la información necesaria para el sistema de prescripción de ejercicio físico.

- Apache: Apache es un servidor web de código abierto multiplataforma que implementa el protocolo de comunicación HTTP/1.1 (Hypertext Transfer Protocol - Protocolo de Transferencia de Hipertextos). Apache tiene amplia aceptación en la red. En el prototipo se usa para atender las peticiones de los especialistas y usuarios, tanto para acceso a los datos como para la comunicación de eventos.

- PHP: (PHP Hypertext Pre-processor) es un lenguaje de programación interpretado de alto nivel, diseñado para el desarrollo web de contenido dinámico. Los scripts PHP son los encargados de los accesos a la base de datos y la comunicación de los eventos.

- JSON: (JavaScript Object Notation - Notación de Objeto JavaScript) es un formato bastante ligero empleado para el intercambio de datos, siendo un subconjunto de la notación para objetos empleada en JavaScript (lenguaje de 
programación muy utilizado en páginas web). En el prototipo se utiliza este formato para el intercambio de los datos entre el servidor y el usuario o el especialista.

- Kafka: Apache Kafka es un sistema de almacenamiento publicador/subscriptor distribuido, particionado y replicado. Estas características, añadidas a que es muy rápido en lecturas y escrituras lo convierten en una herramienta excelente para comunicar eventos que se generan a gran velocidad y que deben ser gestionados por una o varias aplicaciones.

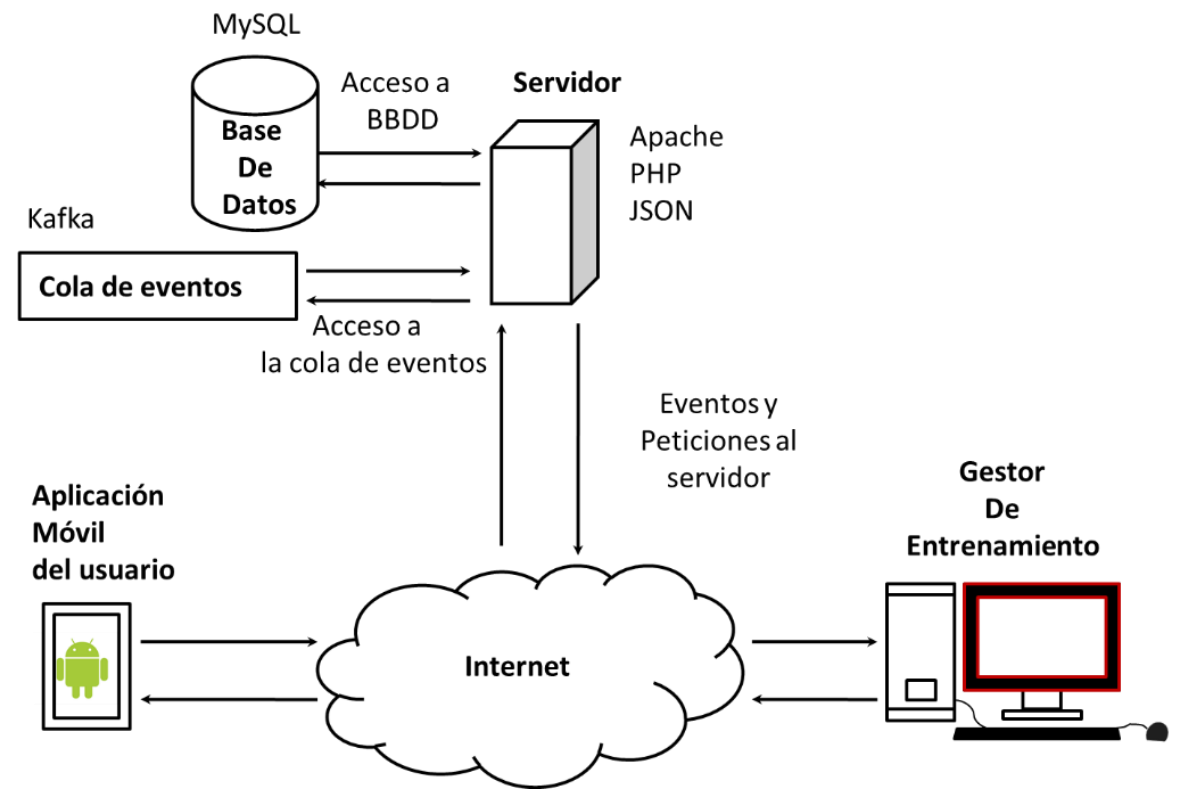

Ilustración 4. Arquitectura del prototipo

Se ha construido una interfaz de usuario amigable, tanto para la aplicación del especialista como para la aplicación móvil del usuario. En la ilustración 5 se puede ver la interfaz de usuario de la aplicación del especialista. En ella se pueden ver cuatro pestañas diferentes, correspondientes a Usuario, Rutinas, Ejercicios y Log. Las tres primeras pestañas se utilizan para la gestión de usuarios, rutinas y ejercicios respectivamente, que permiten la creación, modificación y eliminación de estos. La pestaña de Log permite al especialista visualizar los eventos producidos por los usuarios. En la ilustración 5 también se muestra la pestaña Log de la aplicación. 


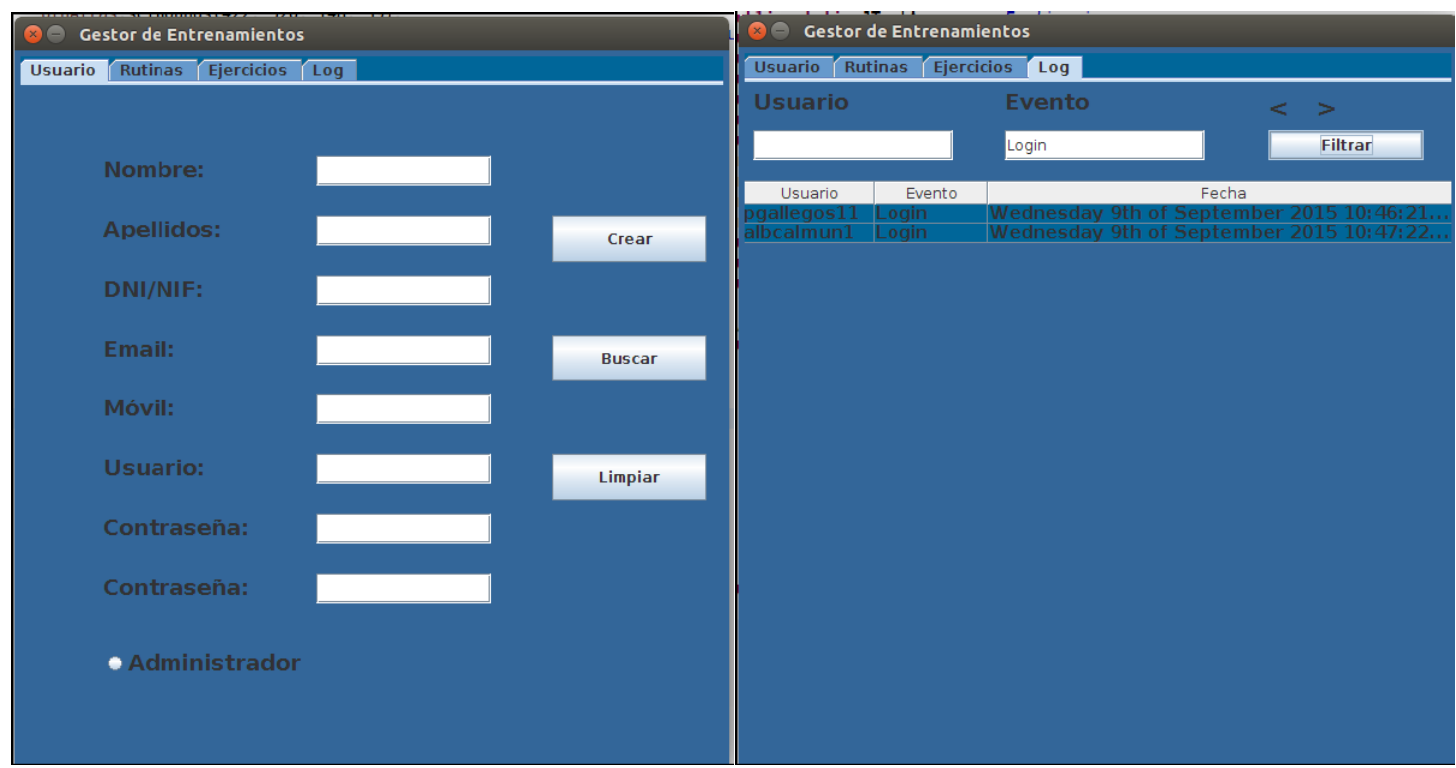

Ilustración 5. Interfaz de la aplicación del especialista

Algunas de las pantallas de la aplicación móvil del usuario se muestran en la ilustración 6. La pantalla que se le presenta al usuario tras la identificación mediante un nombre de usuario y una contraseña, la pantalla que permite al usuario seleccionar los ejercicios de una rutina concreta y la pantalla que permite visualizar la información referente a un ejercicio.

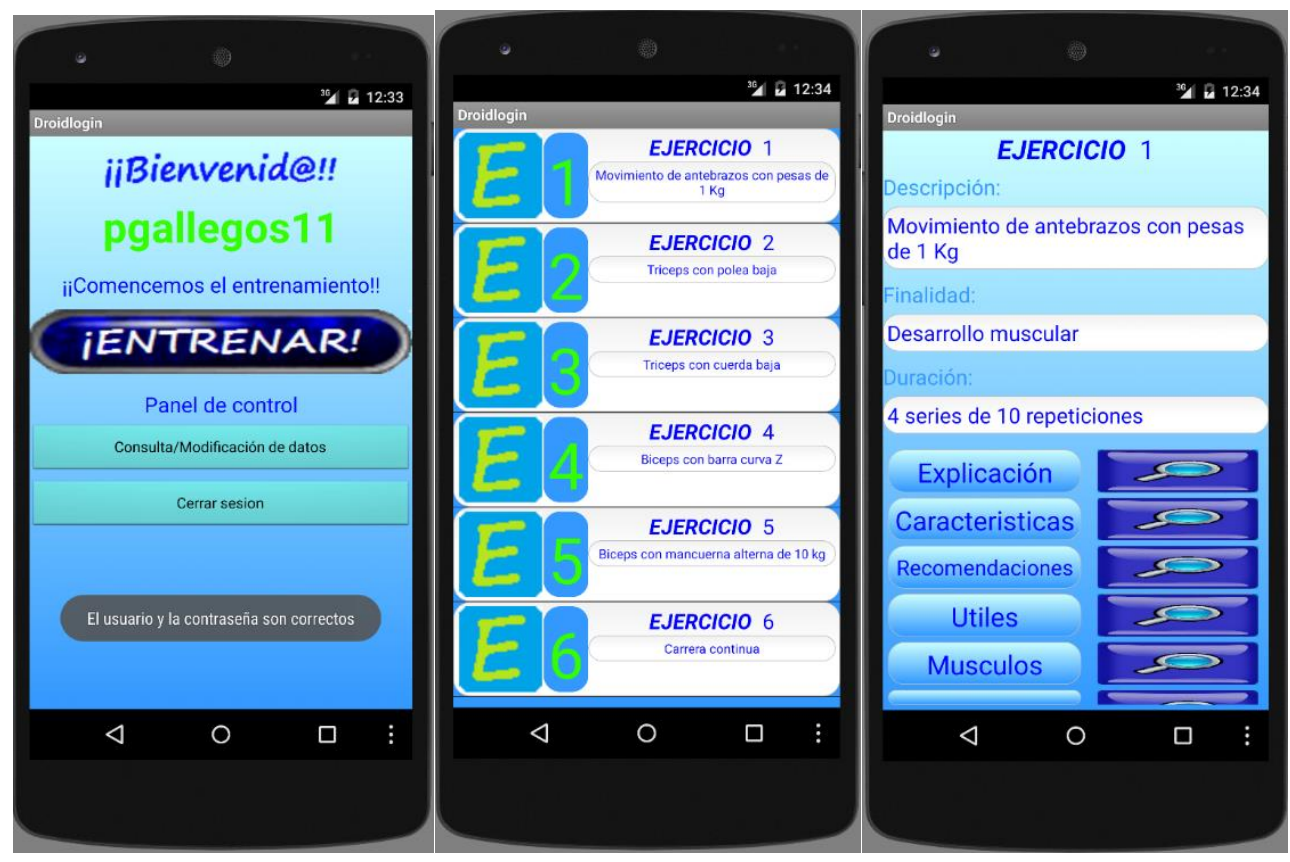

Ilustración 6. Pantallas de la aplicación móvil 


\section{RESULTADOS}

Los resultados obtenidos en este trabajo son por un lado una propuesta de arquitectura software para la mejora de la prescripción de ejercicio físico personalizado y por otra el desarrollo de un prototipo basado en la arquitectura propuesta. Con esta arquitectura, el personal especializado tiene una forma más eficaz de prescribir a sus pacientes los ejercicios que permitan mejorar su condición física, contando con la ayuda de la aplicación móvil en la que el usuario podrá visualizar los ejercicios y las recomendaciones del especialista para realizarlo, sin necesidad de desplazarse ni en horarios prefijados.

\section{DISCUSIÓN Y CONCLUSIONES}

Hoy en día las tecnologías están presentes en todos los aspectos de nuestras vidas y la mejora de las condiciones físicas es un aspecto realmente importante que afecta a la calidad de vida y a la salud. En este trabajo se presenta la arquitectura sofware de una aplicación que permite a un especialista prescribir ejercicio físico personalizado a una determinada persona, adaptado a sus condiciones físicas y sus necesidades. Se ha presentado un prototipo que contempla tanto la parte de la prescripción del especialista como la parte del usuario. Con la ayuda de esta aplicación, el personal especializado podrá indicar los ejercicios a realizar por los usuarios, que contarán con una aplicación móvil para el seguimiento de los ejercicios prescritos.

Debido a que este trabajo se ha centrado en el diseño de la arquitectura y la realización de un prototipo, pueden plantearse diversas mejoras como las mencionadas a continuación. Se podría mejorar la comunicación entre el especialista y el usuario mediante un sistema de mensajería. Se debe ampliar la base de datos de los vídeos de los ejercicios. Se puede incorporar el uso de dispositivos externos que permitan la medición de parámetros físicos del usuario mediante sensores. Utilizar un sistema de seguridad que nos permita autenticar el dispositivo de usuario sin tener que identificar al usuario cada vez que se inicia la aplicación facilitaría el acceso. La aplicación móvil del usuario también se podría programar para el sistema operativo iOS, para llegar a los usuarios que usan este sistema operativo. Para ampliar el número de usuarios que puedan acceder a la aplicación se podría desplegar en la "nube" para que sea escalable.

\section{REFERENCÍAS BIBLIOGRÁFICAS}

1. Sun, F., Norman, I., While, A. (2013). "Physical activity in older people: A systematic review", BMC Public Health, vol. 13, no. 1, pp. 449-465. https://www.ncbi.nlm.nih.gov/pubmed/23648225. DOI: https://doi.org/10.1186/1471-2458-13-449

2. Bonet, J., Parrado, E. y Capdevila, L. (2017). Efectos agudos del ejercicio físico sobre el estado de ánimo y la HRV / Acute Effects of Exercise on Mood And 
HRV. Revista Internacional de Medicina y Ciencias de la Actividad Física y el $\begin{array}{lllll}\text { Deporte. } & \text { vol. } & 17 & \text { (65) } & \text { 85-100. }\end{array}$ http://cdeporte.rediris.es/revista/revista65/artefectos783.htm. DOI: https://doi.org/10.15366/rimcafd2017.65.006

3. Mora-Fernández, M., Mora, V. J., González-Montesinos, J., et al. (2005) Valoración de las mejoras provocadas en la capacidad aeróbica en mujeres sedentarias tras un programa de ejercicios. Revista Internacional de Medicina y Ciencias de la Actividad Física y el Deporte, vol. 5, pp. 39-49.

4. Casajús, J., y Vicente-Rodríguez, G. (2011). Ejercicio físico y salud en poblaciones especiales. 1th ed. Madrid: Exernet, Consejo Superior de Deportes.

5. Márquez, S., y Garatachea, N. (2010). Actividad Física y salud. 1th ed. Madrid: Díaz de Santos, Fundación Iberoamericana.

6. Sañudo, B., Martínez de Haro, V., y Muñoz, J. (2010). Actividad Física en poblaciones especiales. 1th ed. Seville: Wanceulen, Salud y calidad de vida.

7. Poushter, J. (2016). "Smartphone Ownership and Internet Usage Continues to Climb in Emerging Economies", [online] Available: http://www.pewglobal.org/2016/02/22/smartphone-ownership-and-internetusage-continues-to-climb-in-emerging-economies/.

8. Physical Therapy Web. Exercise Prescription Software, http://physicaltherapyweb.com/exercise-prescription-software/ (accessed 26 July 2016).

Número de citas totales / Total references: 8 (100\%)

Número de citas propias de la revista / Journal's own references: 2 (25\%)

Rev.int.med.cienc.act.fís.deporte - vol. 19 - número 73 - ISSN: 1577-0354 